University of Nebraska - Lincoln

DigitalCommons@University of Nebraska - Lincoln

P. F. (Paul Frazer) Williams Publications

Electrical \& Computer Engineering, Department

May 1976

\title{
Comment on "Two-Magnon Resonant Raman Scattering in $\mathrm{MnF}_{2}$ "
}

D. L. Rousseau

Bell Laboratories, Murray Hill, New Jersey

R. E. Dietz

Bell Laboratories, Murray Hill, New Jersey

P. F. Williams

Bell Laboratories, Murray Hill, New Jersey

H. J. Guggenheim

Bell Laboratories, Murray Hill, New Jersey

Follow this and additional works at: https://digitalcommons.unl.edu/elecengwilliams

Part of the Electrical and Computer Engineering Commons

Rousseau, D. L.; Dietz, R. E.; Williams, P. F.; and Guggenheim, H. J., "Comment on "Two-Magnon Resonant Raman Scattering in $\mathrm{MnF}_{2}$ "'" (1976). P. F. (Paul Frazer) Williams Publications. 36.

https://digitalcommons.unl.edu/elecengwilliams/36

This Article is brought to you for free and open access by the Electrical \& Computer Engineering, Department of at DigitalCommons@University of Nebraska - Lincoln. It has been accepted for inclusion in P. F. (Paul Frazer) Williams Publications by an authorized administrator of DigitalCommons@University of Nebraska - Lincoln. 


\title{
Comment on "Two-Magnon Resonant Raman Scattering in $\mathrm{MnF}_{2}$ "
}

\author{
D. L. Rousseau, R. E. Dietz, P. F. Williams,* and H. J. Guggenheim \\ Bell Laboratories, Murray Hill, New Jersey 07974 \\ (Received 4 August 1975; revised manuscript received 22 December 1975)
}

\begin{abstract}
We have not been able to reproduce the recently reported resonance enhancement of Raman scattering from magnons in $\mathrm{MnF}_{2}$ by Amer, Chiang, and Shen. Fifteen crystals ranging from extremely pure samples to ones with high concentrations of impurities, in addition to one supplied by Amer, Chiang, and Shen, were investigated. By observing the off-resonance scattering, we conclude that if an enhancement of greater than a factor of 2 were present, it would have been seen in our resonance spectra.
\end{abstract}

Recently Amer, Chiang, and Shen $(\mathrm{ACS})^{1}$ reported very interesting two-magnon resonanceRaman-scattering spectra from $\mathrm{MnF}_{2}$. They excited $\mathrm{MnF}_{2}$ with a dye laser in the region of the magnon sidebands of the $E_{1}$ and $E_{2}$ excitons and observed the three two-magnon Raman modes, corresponding to the $\sigma_{1}, \pi_{1}$, and $\sigma_{2}$ sidebands, to be resonantly enhanced. We found this experiment particularly intriguing because it may lead both to better understanding of the basic resonance-Raman processes in crystals and to better understanding of the very complex exciton-magnon dynamics in $\mathrm{MnF}_{2}$. On the other hand, it is quite surprising that this very intense Raman scattering was not observed by Dietz et $a l .^{2}$ when they pressure tuned the $\pi_{1}$ sideband through the 5419-A output of a xenon laser, nor was it reported by Macfarlane and Lunt $z^{3}$ in their lifetime experiments in which a dye laser was tuned th rough the $\sigma_{1}$ sideband.

We undertook to study resonant-Raman scattering from $\mathrm{MnF}_{2}$ in the region of the magnon sidebands by illuminating a $\mathrm{MnF}_{2}$ crystal of extremely high purity with the output of a cw dye laser (Rhodamine 110). The laser output power was about $100 \mathrm{~mW}$ throughout the region of interest around the magnon sidebands. Several experi- ments were performed. In some, the beam was focused into crystals which were immersed in superfluid helium at $<2{ }^{\circ} \mathrm{K}$. In others, crystals were placed on the cold finger of an Air Products Heli-Tran cryostat. In this case we estimate the crystal temperature to have been about $10^{\circ} \mathrm{K}$. The scattered light was gathered at $90^{\circ}$ and analyzed with a Spex 1401 double monochromator.

In all of our experiments the utmost care was used to assure that no experimental artifacts were present in our data. Specifically we took the following measures: (1) Careful alignment and aperaturing was done to minimize the amount of dye-laser fluorescence reaching the spectrometer. (2) To be sure that no exciton migration occurred, the diameter of the intrinsic luminescence emanating from the waist of the focused laser beam was measured. It was found to be less than $20 \mu \mathrm{m}$, roughly what we would expect for the width of the dye-laser waist itself. This guaranteed that misalignment effects in which we could have observed intrinsic luminescence but not Raman scattering would be impossible in our experiments. (3) Excitation spectra were run after any change in optical alignment to determine if any thermal-heating effects were occurring at resonance. When such effects were 


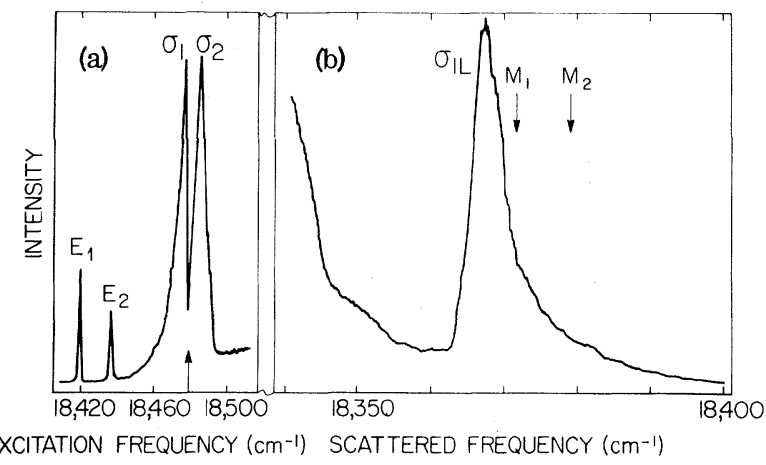

FIG. 1. (a) Excitation spectrum of $\mathrm{MnF}_{2}$ showing the $\sigma_{1}$ and $\sigma_{2}$ sidebands. The arrow indicates the laser frequency used to obtain the scattering spectrum in (b). (b) Scattering spectrum of $\mathrm{MnF}_{2}$ excited at $\sim 18480$ $\mathrm{cm}^{-1}$. The strong feature at $18367 \mathrm{~cm}^{-1}$ is the intrinsic $\sigma_{1 L}$ luminescence. The $M_{1}$ and $M_{2}$ arrows designate the expected frequencies of the two-magnon resonance Raman scattering associated with the $\sigma_{1}$ and $\sigma_{2}$ sidebands, respectively.

seen the laser power was accordingly reduced.

Many crystal orientations were tried in our experiments although emphasis was placed on looking at tensor components expected to exhibit the strongest nonresonant, two-magnon, Raman scattering ${ }^{4}\left(\sigma_{x z}\right.$ and $\left.\sigma_{x y}\right)$. In addition, emphasis was placed on orienting the crystal so as to observe the scattering along the $c$ axis. In this axial orientation $^{2}$ we were able to eliminate nearly completely the $\mathrm{Zn}^{++}$and $\mathrm{Mg}^{++}$impurity exciton lines ${ }^{5}$ at 18383 and $18372 \mathrm{~cm}^{-1}$, respectively, from our spectra.

In Fig. 1(a) we show the fluorescence excitation spectrum of one of our most pure crystals (No. 1 in the Table I). As expected, in the orientation shown here (incident polarization perpendicular to the $c$ axis) we were able to see the $E_{1}$ and $E_{2}$ excitons and the $\sigma_{1}$ and $\sigma_{2}$ sidebands. $^{2}$ By rotating the incident polarization we were able to observe the $\pi_{1}$ sideband. In Fig. 1(b) we present the $X(Y X) Z$ Raman scattering spectrum at $2{ }^{\circ} \mathrm{K}$ with the dye-laser frequency set in the valley between the $\sigma_{1}$ and $\sigma_{2}$ sidebands $\left(\sim 18480 \mathrm{~cm}^{-1}\right)$. This position is designated by the arrow in the excitation spectrum. Note that the $\mathrm{Zn}^{++}$and $\mathrm{Mg}^{++}$ impurity lines are absent from our luminescence spectrum and the only feature is $\sigma_{1 L}$ at 18367 $\mathrm{cm}^{-1}$. The long tail from 18370 to $18400 \mathrm{~cm}^{-1}$ does not result from impurities but is the expected intrinsic $\sigma_{1 L}$-luminescence shape. ${ }^{2}$ For this incident frequency from the data presented in Fig. 3 of Ref. 1, two two-magnon Raman bands are located at the $M_{1}$ and $M_{2}$ positions designated by the arrows. It should be noted that at approximately this same excitation frequency in the report of $\mathrm{ACS}^{1}$ one of the two-magnon resonance Raman lines had a peak intensity of $\sim 50 \%$ of the peak intensity of the $\sigma_{1 L}$ intrinsic luminescence. We investigated many excitation frequencies covering the entire range which they ${ }^{1}$ studied. In no case were we able to observe the two-magnon Raman scattering in the resonance region. Furthermore, because of our crystal orientation and high purity, in our spectra we were able to completely eliminate the $\mathrm{Mg}^{++}$impurity luminescence at $18372 \mathrm{~cm}^{-1}$ which was the strongest feature in the spectra of $\mathrm{ACS}^{1}$ and partially masked the two-

TABLE I. Source, growth method, and total impurity content of crystals studied from emission spectrographic analysis.

\begin{tabular}{clll}
\hline \hline Crystal & Source & Growth method & \multicolumn{1}{c}{$\begin{array}{c}\text { Total impurities } \\
\text { (ppm) }\end{array}$} \\
\hline 1 & BTL & Horizontal zone & $<300$ \\
2 & Stanford & Unknown & $<300$ \\
3 & BTL & Horizontal zone & $<300$ \\
4 & BTL & Unknown & $<300$ \\
5 & BTL & Bridgeman & $<300$ \\
6 & BTL & Horizontal zone & $<400$ \\
7 & BTL & Horizontal zone & $<400$ \\
8 & BTL & Horizontal zone & $<400$ \\
9 & BTL & Bridgeman & $<400$ \\
10 & BTL & Horizontal zone & $10^{2}-10^{3} \mathrm{Si}, \mathrm{Ag} ;<300$ other metals \\
11 & BTL & Unknown & $10^{3}-10^{4} \mathrm{Mg}, \mathrm{Fe} ;<300$ other metals \\
12 & BTL & Unknown & $10^{2}-10^{3} \mathrm{Ni}<400$ other metals \\
13 & BTL & Horizontal zone & $10^{2}-10^{3} \mathrm{Ni}$; $10^{3}-10^{4}$ Co; $<400$ other metals \\
14 & BTL & Horizontal zone & $10^{2}-10^{3} \mathrm{Ca} ;<200$ other metals \\
\hline
\end{tabular}


magnon Raman lines. We would therefore expect to have greater sensitivity than theirs for detecting these Raman lines.

To determine whether or not this first crystal was unique in its failure to exhibit the reported resonance spectra, we investigated several other crystals. All of our crystals with their method of growth and impurity content are listed in Table I. The impurity contents listed in Table I are upper limits of the total cation impurities based on emission spectrographic analysis and confirmed by secondary ion mass spectrometry and spark-source mass spectroscopy. For the purest crystals we believe a realistic estimate of the total cation impurity level to be in the range of $100 \mathrm{ppm}$. In the excitation spectrum of some of the crystals with high impurity concentrations we observed either a splitting or a substantial broadening of the intrinsic exciton and magnon sideband lines. However in none of the crystals were we able to see the two-magnon resonanceRaman scattering. Recently we have exchanged crystals with ACS. We found that their crystal exhibited several strong impurity lines. As in our crystals we were unable to observe any resonance enhancement of the two-magnon Raman scattering.

To determine the expected intensity of the twomagnon Raman scattering in the resonance region we investigated an additional crystal at $\sim 10^{\circ} \mathrm{K}$ cut from the same boule as crystal No. 1. In this series of experiments we repeated the off-resonance Raman measurements of Fleury, Porto, and Loudon ${ }^{4}$ with 4880 - $\AA$ excitation and then using the discrete lines of argon and krypton lasers we followed the Raman scattering to the 5308- $\AA$ krypton laser excitation which overlapped the wavelength range of the dye laser. The Raman scattering was then followed as the dye laser was tuned into resonance. In Fig. 2 we show two of these spectra when the excitation is near resonance with $\sigma_{2} . M$ is the two-magnon Raman scattering. For $18524 \mathrm{~cm}^{-1}$ excitation the two-magnon scattering lies to the high-energy side of the $E 1$ exciton, while at $18506 \mathrm{~cm}^{-1}$ it has moved into the wing of the magnon sideband. As the excitation frequency was moved further into resonance with $\sigma_{2}$ the two-magnon Raman scattering became lost in luminescence from the sharply sloping magnon sideband.

$\mathrm{ACS}^{1}$ reported a factor of 7 enhancement of the two-magnon Raman scattering at resonance with the $\sigma_{2}$ sideband. In addition they reported that in the resonance region the two-magnon line-

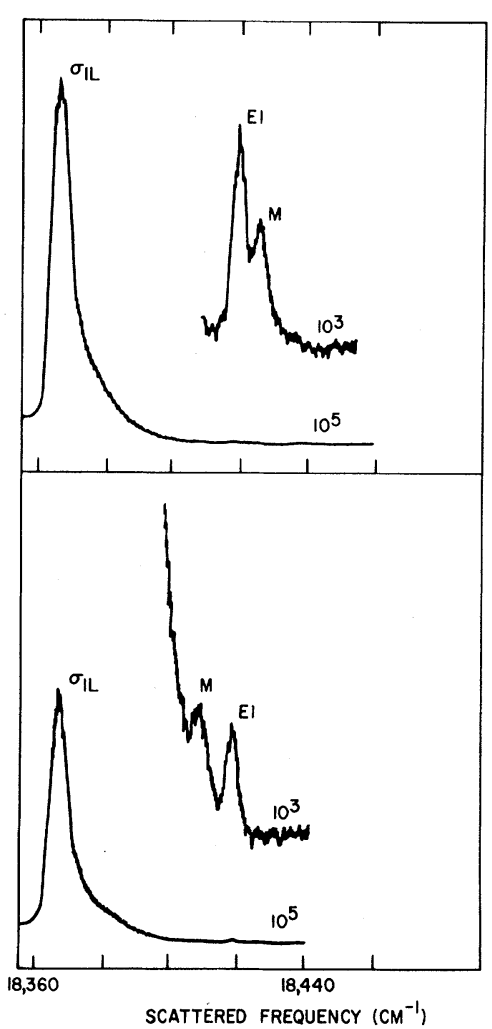

FIG. 2. Re-emission spectrum from $\mathrm{MnF}_{2}$ slightly away from resonance with the $\sigma_{2}$ sideband. The $10^{3}$ and $10^{5}$ refer to counts per second full scale. $E 1, \sigma_{1 L}$, and $M$ refer to emission from the $E 1$ exciton, its magnon sideband, and the two-magnon Raman scattering, respectively. The top spectrum was obtained with $18524-\mathrm{cm}^{-1}$ excitation and the bottom with $18506-\mathrm{cm}^{-1}$ excitation.

width was instrumentally limited. To determine the limits of our sensitivity for detecting the twomagnon resonance Raman scattering we measured the re-emission with excitation of about 18530 $\mathrm{cm}^{-1}$ so that both the $E 1$ emission with an instrumentally limited width and the off-resonance Raman emission were resolved and observed in the same spectrum. These data were stored on one quadrant of a multichannel analyzer. The integrated intensity of the exciton was adjusted to equal that of the Raman scattering. The excitation frequency was then tuned to the peak of $\sigma_{2}$ and a spectrum was obtained [it appeared qualitatively similar to that shown in Fig. 1(a)] and stored in a different quadrant of the analyzer. The positions of the two spectra were adjusted such that the $E 1$ exciton line in the first spectrum coincided with the expected position of the twomagnon resonance Raman scattering in the sec- 
ond spectrum. By adding the two spectra and varying their relative intensities we then were able to determine the level of enhancement observable on the $\sigma_{1 L}$-luminescence background in our experiments. From these measurements a factor of 7 enhancement, as reported by $\mathrm{ACS}^{1}$ would be very readily observable in our data. Indeed if the enhancement were as small as a factor of 2 we would have been able to observe it.

The contrast between our results and those of $\mathrm{ACS}^{1}$ is striking. They report an enhancement of an order of magnitude and a peak intensity of the resonance Raman scattering comparable to that of $\sigma_{1} x$. Any possible enhancement in our crystals is less than a factor of 2 and the total Raman two-magnon intensity is down from $\sigma_{1 L}$ by more than two orders of magnitude. We have examined several crystals ranging from some which we believe to be among the purest available to others which have high concentrations of impurities, hoping to determine if a certain impurity level was responsible for the scattering data reported by $\mathrm{ACS},{ }^{1}$ but in no cases were we able to observe any enhancement.

Aside from our inability to reproduce the resonantly enhanced Raman spectra of ACS, ${ }^{1}$ we believe there are other strong grounds for rejecting the validity of their claims. Most importantly, they neglect the strong exciton-magnon interaction which was shown by Dietz et $a l_{\circ}^{2}$ to be solely responsible for the difference in shapes between $\sigma_{1}$ and $\sigma_{2}$. This was demonstrated by showing that the magnon sidebands of $E 1$ and $E 2$ have different shapes only in an absorption process when the exciton and magnon are created simultaneously. In fluorescence, the exciton is annihilated with the subsequent creation of a mag- non, with the result that the re is no strong interaction, and consequently both sidebands have the same shape, indicating that $E 1$ and $E 2$ have identical dispersions. A calculation of the emission sideband shape ${ }^{6}$ using neutron-scattering parameters ${ }^{7}$ for the magnon dispersion demonstrated that both $E 1$ and $E 2$ had less than $0.5 \mathrm{~cm}^{-1}$ dispersion, contrary to the dispersion of $7 \mathrm{~cm}^{-1}$ assumed for $E 2$ in the analysis by ACS. ${ }^{1}$ The effect of a dispersionless $E 2$ exciton would be to shift the position of the $\sigma_{2}$ resonance well outside the range of their experimental uncertainty. Appropriate treatment of the exciton-magnon and magnon-magnon interaction would significantly broaden $\left(5-10 \mathrm{~cm}^{-1}\right)$ the resonance Raman scattering.

In view of the serious ir reproducibility of the results of ACS coupled with the theoretical objections raised above, we believe the differences between our results and theirs must lie in some experimental artifact in their data.

* Present address: Department of Physics, University of Puerto Rico, Rio Piedras, Puerto Rico 00931.

${ }^{1}$ N. M. Amer, T.-c. Chiang, and Y. R. Shen, Phys. Rev. Lett. 34, 1454 (1975).

${ }^{2}$ R. E. Dietz, A. E. Meizner, H. J. Guggenheim, and A. Misetich, J. Lumin. 1,2, 279 (1970).

${ }^{3}$ R. M. Macfarlane and A. C. Luntz, Phys. Rev. Lett. 31, 832 (1973).

${ }^{4}$ P. A. Fleury, S. P. S. Porto, and R. Loudon, Phys. Rev。 Lett. 18, 658 (1967).

${ }^{5}$ A. Misetich, R. E. Dietz, and H. J. Guggenheim, Localized Excitations in Solids (Plenum, New York, 1968), p. 379.

${ }^{6}$ R. E. Dietz, A. E. Meizner, H. J. Guggenheim, and A. Misetich, Phys. Rev. Lett. 21, 1067 (1968) .

${ }^{7}$ G. G. Low, A. Okazaki, K. C. Turberfield, and R. W. H.Stevenson, Phys. Lett. $\underline{8}, 9$ (1964). 